\title{
Monoclonal gammopathy of renal significance - diagnostic and therapeutic problems
}

\section{Grzegorz Kade', Sebastian Spaleniak', Janusz Hałka ${ }^{3}$, Stefan Antosiewicz}

${ }^{1}$ Warmian-Masurian Cancer Center of the Ministry of the Interior and Administration's Hospital in Olsztyn, Poland

${ }^{2}$ Department of Internal Diseases and Nephrodiabetology, Medical University of Lodz

${ }^{3}$ Department of Clinical Hematology, Warmian-Masurian Cancer Center of the Ministry of the Interior and Administration's Hospital, Poland

${ }^{4}$ Military Institute of Aviation Medicine, Center of Aeromedical Examination and Occupational Medicine, Poland

Correspondence:

Grzegorz Kade

Warmian-Masurian Cancer Center of the Ministry of the Interior and Administration's

Hospital in Olsztyn, Poland 10-228 Olsztyn, Wojska Polskiego 37 phone: 895398000

fax: 895398240

e-mail:grzegorz.kade@poliklinika.net

Received:

4.01.2021

Accepted:

30.01.2021

DOI: 10.24292/01.OR.420300121 Copyright $\odot$ Medical Education. All rights reserved.

\section{ABSTRACT}

The term monoclonal gammopathy of renal significance MGRS means a group of renal diseases resulting from the presence of the monoclonal protein produced by plasmatic cells or other clones of B cells. The patients with MGRS do not fulfill the diagnostics criteria both of multiple myeloma and other neoplasm originating from B cells. The involvement of different renal structures in the course of MGRS results the dysfunction of kidneys. The monoclonal protein may injure the glomerular structures (including vascular) as well as tubular structures (interstitial in more wide sense). The early diagnosis of MGRS is difficult and the late detection of the disease is connected with high risk of irreversible renal damage. Therefore, the multidisciplinary cooperation - including general practitioners, nephrologists, hematologists and nephro-pathologists - is particularly important for the diagnostics and treatment of MGRS cases. This new hemato-nephrological meta-disease entity is connected with relatively high morbidity and mortality as well as relapses in transplanted kidney. The decision of the treatment initiation against the toxic clone in MGRS cases results mainly from the nephrological reasons. The article presents current diagnostic and therapeutic possibilities that may be used in MGRS patients. The main purpose of this article was to present the current state of knowledge regarding the diagnostics and treatment of MGRS.

Key words: monoclonal gammopathy of renal significance, monoclonal gammopathy of undetermined significance, kidney disease 


\section{INTRODUCTION}

The renal damage resulting from nephrotoxicity of monoclonal protein (immunoglobuline or its part) being the monoclonal gammopathy product and fulfilling criteria of MGUS (monoclonal gammopathy of undetermined significance) is characteristic for MGRS (monoclonal gammopathy of renal significance). The MGRS cases from the definition do not fulfill the criteria of multiple myeloma nor B-cell lymphoma. MGRS term does not include many diseases caused by lymphoproliferative disorders of high malignance grade. The pathogenicity of produced monoclonal protein in MGRS, causes the damage of different renal structures leading to the end-stage insufficiency in some cases.

\section{MONOCLONAL GAMMOPATHY OF UNDETERMINED SIGNIFICANCE - DEFINITION AND DIAGNOSTIC CRITERIA}

The group of experts participating in the activity of International Kidney and Monoclonal Gammopathy Reseach Group (IKMG) introduced in 2012 the term monoclonal gammopathy of renal significance (MGRS). The mentioned above group of experts comprising nephrologists, hematologists and nephro-pathologists from different countries was founded in 2010 [1]. The IKMG foundation was inspired by the dilemma connected with treatment of patients with renal diseases caused by monoclonal proteins. Lack of therapeutic guidance on cytotoxic treatment in this group of patient leaded to high morbidity (mainly due to renal insufficiency) and even high mortality. MGRS comprises all disorders of the renal function induced by monoclonal immunoglobuline or its parts produced by non-malignant clone of B lymphocytes. The early diagnosis of MGRS is particularly important because it gives the chance for the early introduction of the proper therapy inhibiting the production of monoclonal proteins. The IKMG work resulted in the clear distinction between benign hematological disorders which do not lead to the renal damage (MGUS) and MGRS which is connected with the deposition of monoclonal immunoglobuline or its part in kidneys that is the cause of higher morbidity. We should also understand that both MGUS and MGRS do not exclude the progression to the defined hematological disease. It is commonly known that the incidence of monoclonal gammopathy increases with age and therefore the majority of cases is recognized in persons in the sixth decade of life or older. The main age of patients with MGRS diagnosed using renal biopsy is $62,5 \pm 9,1$ years [2].

In April 2017 IKMG gathered again to clarify the MGRS definition and to update the diagnostic criteria of MGRS related diseases. MGRS was defined as a clonal proliferative disorder that produc- es a nephrotoxic monoclonal immunoglobulin and does not meet hematological diagnostic criteria of a specific malignancy. The role of renal biopsy in MGRS diagnose and optimal therapy choice was underlined in the IKMG guidelines. Furthermore, the flow cytometry was mentioned as potentially useful method of small clones identification [3]. Steiner et al. found 44 cases of MGRS $(1,5 \%)$ in the group of 2935 patients with MGUS observed in the years 2000-2016. The progression to plasma cell myeloma was significantly more frequent in MGRS patients than in MGUS $(18 \%$ vs $3 \% ; p<0,001)$ [4]. The presence of one class serum monoclonal protein - $\lg \mathrm{G}, \lg \mathrm{A}$, $\lg \mathrm{M}$ in a concentration lower than $30 \mathrm{~g} / \mathrm{l}$, less than $10 \%$ of monoclonal bone marrow plasma cells and lack of organ damage caused by gammopathy are observed in MGUS cases. The organ damage is described by SLIM CRAB acronym. SLiM means accordingly:

- S (sixty) - more than $60 \%$ of clonal plasma cells in bone marrow or tissue biopsy

- Li (light chains) - clonal to non-clonal free light-chain ratio in serum of 100 or higher (the clonal light chain serum concentration not lower than $100 \mathrm{mg} / \mathrm{l})$

- $\mathrm{M}$ (magnetic resonance) - presence of more than one bone lesion on MRI, bigger than $5 \mathrm{~mm}$.

\section{CRAB abbreviation means:}

- hypercalcaemia, renal impairment, anaemia and lytic bone lesions

- C (calcium) - calcium concentration 0,25 mmol/l (1 mg/dl) higher than upper reference value or higher than $2,75 \mathrm{mmol} / \mathrm{l}(>11 \mathrm{mg} / \mathrm{dl})$

- $\mathrm{R}$ (renal insufficiency) - serum creatinine higher than $177 \mu \mathrm{mol} / \mathrm{l}$ (> $2 \mathrm{mg} / \mathrm{dl}$ ) or creatinine clearance $<40 \mathrm{ml} / \mathrm{min}$ (measured or calculated)

- A (anemia) - serum hemoglobin $2 \mathrm{~g} / \mathrm{dl}$ lower than lower reference value or lower than $10 \mathrm{~g} / \mathrm{dl}$ and

- B (bones) - one or more osteolytic lesion revealed in classic radiogram, $\mathrm{CT}$ or $\mathrm{PET}-\mathrm{CT}$.

MGUS is detected in about $5 \%$ of persons aged $>70$ years, male more frequent than female. The development of multiple myeloma in approximately $80 \%$ of cases takes place due to evolution of MGUS other than IgM (non-IgM MGUS) and in $20 \%$ of cases light-chain immunoglobulin MGUS - LC-MGUS [5]. The efficient and quick diagnostics is crucial for the prognosis in MGRS cases. In the Polish medical care system, general practitioners may order the following laboratory tests: blood count with differential count, basic coagulation tests, biochemical tests, electrophoresis of serum proteins, erythrocyte sedimentation rate, urinalysis, 24-hours urine collection and proteinuria assessment and fecal 
occult blood test. Furthermore, family doctors may order chest, skull, spine, upper/lower extremities X-rays, as well as ultrasonography of abdomen and peripheral lymph nodes. They are also authorized to order ECG, gastroscopy and colonoscopy. Endoscopic examination is particularly important for AL amyloidosis diagnostics due to the fact that they enable taking the mucosa specimen. The renal biopsy is particularly important for the diagnostics of the nephropathy related to MGRS [7]. The nephro-pathology assessment includes light microscopy, immunofluorescence and electron microscopy. In some cases it is necessary to perform the immune electron microscopy. Some authors propose to use proteomic analysis in selected cases [8]. The abnormalities observed in MGRS renal biopsy are caused by two main reasons. First of them results from the deposition of immunoglobulines or immunoglobuline parts in the renal structures. The second is related to the activity of monoclonal proteins as anti-complement auto-antibodies or anti-A2 phospholipase receptors antibodies. The protein deposition in different renal structures and in different ways does not result from the quantity of protein but is related to its structure and physico-chemical properties. It is considered that the sequence of light chain aminoacids determines the tendency to nephropathy occurrence.

The lambda light chains coded by V $\lambda 6 a$ gene cause the glomeruli structure damage probably by the reaction with mesangial cells receptor [10]. One should remember that lack of detection of one of light chains in immunofluorescence test does not exclude the presence of AL amyloidosis. In such cases the mass spectrometry may be useful $[11,12]$. The expression of glomeruli involvement is the renal biopsy may take place as organized or non-organized deposites. In the first case we may observe fibrillary glomerulonephritis (FGN), immunotactoid "rod-like-structures tactoids" glomerulonephritis (GOMMID), type I cryoglobulinemic glomerulonephritis, and light chain amyloidosis (AL), less frequently heavy chain amyloidosis ( $\mathrm{AH}$ ) or $\mathrm{AL}$ and $\mathrm{AH}$ simultaneously. In the last case, the pathological changes may involve also tubuli and blood vessels. The following types of glomerular changes are caused by amorphous deposits: monoclonal immunoglobulin deposition disease (MIDD), proliferative glomerulonephritis with monoclonal IgG depositis (PGNMID), C3 glomerulopathy with monoclonal gammopathy. The pathological changes may involve proximal tubuli and - secondary also parenchyma. The following tubular pathologies may occur: proximal tubulopathy with Fanconi syndrome caused by light chains, proximal tubulopathy without lesions and crystal storing histiocytosis [13, 14].

\section{TREATMENT OF MONOCLONAL GAMMOPATHY OF UNDETERMINED SIGNIFICANCE - GENERAL ISSUES}

The current MGRS therapeutic schedules are based on the treatment targeted in proper pathological B cells clones. These therapeutic schedules are also extrapolation of treatment used in equivalent overt neoplasia. The assessment of renal pathology is very important for the choice of adequate MGRS therapeutic schedule. In particular it comprises the risk of renal disease progression as well as the presence and progression of non-renal symptoms. The optimal choice of pharmaceuticals and doses is very important in renal insufficiency. In early diagnosed MGRS cases, when renal function is normal or close to normal, we expect better long-term results. The MGRS therapy should be performed by highly-specialized centers disposing different therapeutic options prescribed by experienced teems of nephrologists, hematologists and nephro-pathologists, which results from the fact that it is a heterogenic and relatively rare disease. According to the recommendations of International Kidney and Monoclonal Gammopathy Research Group, the chemotherapy should be considered in patients with chronic kidney disease stage I-III to slow the disease progression to end-stage renal disease requiring renal replacement therapy. The available therapeutic schedules are based on cyclophosphamide, bortezomid, or thalidomide or bendamustine. High doses of melphalan with autologous bone marrow transplantation may be considered in some cases with poor response to other therapeutic schedules. The implementation of chemotherapy - including the treatment with melphalan, should be considered in patients with chronic kidney disease - stage IV-V, who may be qualified to renal allo-transplantation. No chemotherapy benefits were observed in patients who are not candidates for kidney transplantation [15].

Proteasome inhibitors - including bortezomib - are the leading MGRS pharmaceuticals according to the most recent guidelines [16]. Bortezomib reduces the proteinuria and inhibits the glomerular damage in course of MGRS due to interruption of cascade of signal transduction pathways such as NF-KB [17]. In the number of publications it was considered as first choice pharmaceutical in MGRS due to availability and relatively good safety profile $[18,19]$. Additionally bortezomib is perfectly suitable for induction treatment prior to autologous stem cell transplantation (ASCT) which is justified by the improved effectiveness of such therapeutic option [20].

One of the most recent MGRS therapeutic schedules is based on daratumumab - the new human monoclonal $\mathrm{CD}_{38}$ receptor-binding antibody. Initially it was registered for a treatment of reverse/resistant to treatment multiple myeloma. Its benefi- 
cial safety profile and higher efficiency in comparison to other medicines caused that daratumumab was introduced for standard treatment of plasmatic myeloma. The clinical trials of daratumumab application in other diseases - such as smoldeuring myeloma, MGUS, MGRS and amyloidosis were also performed [21]. The daratumumab was admnistered in 25 MGRS patients, including 12 patients who did not receive any earlier treatment. The median of observation in this group was 14 months. The complete response to chemotherapy was achieved in five patients (22\%), very good partial response in the next five patients (22\%), partial response in seven patients (30\%) with general response rate $74 \%$. Two of five patients from complete response subgroup and presence of clones at the beginning of therapy demonstrated the absence of minimal residual disease. Daratumumab-based therapy was recognized as a new treatment option for MGRS patients [21, 22]. The therapeutic decisions in MGRS patients with end-stage renal insufficiency requiring kidney transplantation and with relapse - are particularly difficult Leung and Dingli described successful application of venetoclax in patient with amyloidosis relapse, after ineffective treatment with bortezomib and daratumumab [23].

\section{MGRS THERAPEUTIC SCHEDULES ADJUSTED TO KIDNEY PATHOLOGY}

Systemic amyloidosis AL, AH, AHL seems to be an important therapeutic problem. The main purpose of the treatment is to eliminate the plasmatic cells clone responsible for the production of amyloid protein. The therapeutic schedules of light chain amyloidosis are based on cyclophosphamide, bortezomib, and dexamethasone (CyBorD). In the next stage of therapy the application of autologous stem cell transplantation preceded by high dose of melphalan should be considered. ASCT may be considered in young patients with early stage amyloidosis according to Mayo classification - that means I or II stage [24]. In ANDROMEDA clinical trial the effectiveness of daratumumab-CyBorD therapeutic schedule versus CyBorD was analysed in 28 patients with AL amyloidosis. Daratumumab was administered subcutaneously (DARA SC) according to the following schedule: every week in $1^{\text {st }}$ and $2^{\text {nd }}$ cycle, every 2 weeks in cycles 3-6 and then every four weeks for the period of 2 years. CyBorD was administered every once weekly in six cycles. The results of the clinical trial indicated that the schedule including DARA SC is a promising therapeutic option in AL amyloidosis. The general rate of hematologic response was $96 \%$ including $54 \%$ (15 patients) with complete hematologic response [25].
Fibrillary glomerulonephritis (FGN) is a rare primary glomerulonephritis. In the largest study dedicated to FGN, comprising 66 patients, the different therapeutic schedules were used. These schedules included nephroprotection, cyclophosphamide, mycophenolate, lenalidomide, azathioprine and glucocorticosteroids. The prognosis is poor despite the use of different treatment regimens and in approximately $50 \%$ of patients the end-stage renal disease is observed [26].

The treatment of immunotactoid glomerulopathy (ITG) was initially based on corticosteroids combined with cyclophosphamide or melphalan, chlorambucil only or cyclophosphamide with vincristine or with doxorubicin [27]. However, the recent recommendations indicate the benefits of therapeutic schedules with bortezomib, cyclophosphamide and/or bendamusitne and glucocorticosteroids. In the cases of ITG in course of chronic lymphotic leukemia the use of rituximab may be beneficial for patients [6].

In MGRS cases in course of crioglobulinemia type 1 the treatment should be focused on the primary case of the disease. Patients with chronic renal disease and plasma cell dyscrasia should be treated with bortezomib, cyclophosphamide and/or thalidomide. ASCT is one of therapeutic options in multiple myeloma. Rituximab is recommended in cases of presence of $B$ cells clones with CD20 antigen - causing lymphoplasmacytic hyperplasia $[6,13]$.

In MIDD and chronic renal disease stage I-III the therapy should be based on bortezomib. ASCT should be considered when general condition of patient is good. In CKD stage IV-V patient should be qualified for renal transplantation, therapy based on bortezomib and then ASCT. When patient is not eligible for kidney transplantation the therapy is based on bortezomib [15].

The decision to start MGRS treatment may be a challenge - especially in case of undetectable dysproteinemia or lack of evidence of bone marrow clonal disease. The proliferative glomerulonephritis with monoclonal IgG deposits has one of the lowest disproteinemia detection rate in the group of renal diseases with monoclonal IgG deposition. The serum monoclonal protein was detected only in 5 out of the group of 10 patients with PGNMID described by Nasr et al. [28]. Another cohort study including 37 patients with PGNMID revealed that only 10 of patients demonstrated dysproteinemia when the diagnosis was made [29]. PGMID is a rare type of proliferative glomerulonephritis classified as MGRS. In cases of chronic renal disease stage I-II with proteinuria lower than $1 \mathrm{~g} / 24 \mathrm{~h}$, the therapy is limited to ne- 
phroprotection. The chemotherapy with ASCT or without ASCT should be considered when the proteinuria exceeds $1 \mathrm{~g} / 24 \mathrm{~h}$ or renal insufficiency reaches stage II-IV. Patient may be qualified to renal transplantation, chemotherapy and then ASCT in the stage $\mathrm{V}$ of chronic renal disease. The chemotherapy based on cyclophosphamide, bortezomib, dexamethasone and administered prior to renal transplantation may be beneficial to the patient. The therapeutic schedules based on rituximab may be used in PGMID caused by the proliferation of B-cell clones [30, 31].

C3 glomerulopathy with monoclonal gammopathy is the disease resulting from disorders of complement activation alternative pathway. The presence of monoclonal immunoglobuline in serum was confirmed in 10 patients out of the group of 32 patients with C3GN observed in Mayo Clinic. They were treated with different therapeutic schedules - including prednisone only or combined with immunosuppressive drug such as azathioprine, cyclophosphamide or mycophenolate. In one patient the therapeutic schedule with dexamethasone and bortezomib was administered with improvement. Another patient with chronic lymphocytic leukemia was treated with six cycles comprising rituximab, cyclophosphamide, vincristine and prednisone (R-CBVP) [32].

The clinical and histopathological data suggest also a good therapeutic response to eculizumab (monoclonal antibody against the $\mathrm{C} 5$ complement component) in cases of patients with relapsing C3 glomerulopathy caused by monoclonal gammopathy after allogenic renal transplantation [33].

In LCPT and chronic renal disease stage I-III the chemotherapy is based on bortezomib, cyclophosphamide, thalidomide and bendamustine. If the chemotherapy is not effective the ASCT may be considered. In CKD stage IV-V patient should be qualified for renal transplantation, therapy based on bortezomib and then ASCT. When patient is not eligible for kidney transplantation the symptomatic/supportive therapy is administered [14].

\section{CONCLUSION}

MGRS is relatively young disease entity. It is not easy to perform the diagnostics in MGRS suspected cases - it requires not only availability of numerous diagnostic techniques, but also multidisciplinary cooperation - including general practitioners, nephrologists, hematologists and nephro-pathologists. The development of highly-specialized centers with experienced medical personnel and adequate diagnostic methods may contribute to significant reduction of MGRS morbidity and mortality. These centers should have access to modern therapies recommended on the base of clinical trials.

\section{References}

1. Leung $\mathrm{N}$, Bridoux $\mathrm{F}$, Hutchison $\mathrm{CA}$ et al. Monoclonal gammopathy of renal significance: when MGUS is no longer undetermined or insignificant. Blood. 2012; 120(22): 4292-5. http://doi.org/10.1182/blood-2012-07-445304.

2. Menè P, De Alexandris L, Moioli A et al. Monoclonal Gammopathies of Renal Significance: Renal Biopsy and Beyond. Cancers (Basel). 2020; 12(7): 1741. http://doi.org/10.3390/cancers12071741.

3. Leung $\mathrm{N}$, Bridoux $\mathrm{F}$, Batuman $\mathrm{V}$ et al. The evaluation of monoclonal gammopathy of renal significance: a consensus report of the International Kidney and Monoclonal Gammopathy Research Group. Nat Rev Nephrol. 2019; 15(1): 45-59. http://doi.org/10.1038/s41581-018-0077-4. Erratum in: Nat Rev Nephrol. 2019; 15(2): 121.

4. Steiner N, Göbel G, Suchecki P et al. Monoclonal gammopathy of renal significance (MGRS) increases the risk for progression to multiple myeloma: an observational study of 2935 MGUS patients. Oncotarget. 2017; 9(2): 2344-56. http://doi.org/10.18632/oncotarget.23412.

5. Giannopoulos K, Jamroziak K, Usnarska-Zubkiewicz L et al. Zalecenia Polskiej Grupy Szpiczakowej dotyczące rozpoznawania i leczenia szpiczaka plazmocytowego oraz innych dyskrazji plazmocytowych na rok 2018/2019. Acta Acta Haematologica Polonica. 2018; 49(4): 157-206.

6. Jain A, Haynes R, Kothari J et al. Pathophysiology and management of monoclonal gammopathy of renal significance. Blood Adv. 2019; 3(15): 2409-23. http://doi.org/10.1182/bloodadvances.2019031914.

7. Fish R, Pinney J, Jain P et al. The incidence of major hemorrhagic complications after renal biopsies in patients with monoclonal gammopathies. Clin J Am Soc Nephrol. 2010; 5(11): 1977-80. http://doi.org/10.2215/CJN.00650110.

8. Bridoux F, Leung N, Hutchison CA et al. Diagnosis of monoclonal gammopathy of renal significance. Kidney Int. 2015; 87(4): 698-711. http://doi. org/10.1038/ki.2014.408.

9. Ciocchini M, Arbelbide J, Musso CG. Monoclonal gammopathy of renal significance (MGRS): the characteristics and significance of a new meta-entity. Int Urol Nephrol. 2017; 49(12): 2171-5. http://doi.org/10.1007/s11255-017-1594-y.

10. Usnarska-Zubkiewicz L, Dębski J, Kuliczkowski K. Jak obecnie leczyć chorego na szpiczaka mnogiego z niewydolnością nerek? Acta Haematologica Polonica. 2011; 42(2): 215-25.

11. Spodzieja M, Rodziewicz-Motowidło S, Szymanska A. Hyphenated Mass Spectrometry Techniques in the Diagnosis of Amyloidosis. Curr Med Chem. 2019; 26(1): 104-20. http://doi.org/10.2174/0929867324666171003113019.

12. Sethi S, Theis JD, Leung $\mathrm{N}$ et al. Mass spectrometry-based proteomic diagnosis of renal immunoglobulin heavy chain amyloidosis. Clin J Am Soc Nephrol. 2010; 5(12): 2180-7. http://doi.org/10.2215/CJN.02890310. Epub 2010.

13. Skwierawska K, Waszczuk-Gajda A, Perkowska-Ptasińska A et al. Gammapatie monoklonalne o znaczeniu nerkowym. Acta Haematologica Polonica. 2018; 49(2): 50-8. 
14. Jain A, Haynes R, Kothari J et al. Pathophysiology and management of monoclonal gammopathy of renal significance. Blood Adv. 2019; 3(15): 2409-23. http://doi.org/10.1182/bloodadvances.2019031914.

15. Fermand JP, Bridoux F, Kyle RA et al. International Kidney and Monoclonal Gammopathy Research Group. How I treat monoclonal gammopathy of renal significance (MGRS). Blood. 2013; 122(22): 3583-90. http://doi.org/10.1182/blood-2013-05-495929. Epub 2013.

16. Amaador K, Peeters H, Minnema MC et al. Monoclonal gammopathy of renal significance (MGRS) histopathologic classification, diagnostic workup, and therapeutic options. Neth J Med. 2019; 77(7): 243-54.

17. Gandolfi S, Laubach JP, Hideshima T et al. The proteasome and proteasome inhibitors in multiple myeloma. Cancer Metastasis Rev. 2017; 36(4): 561-84. http://doi.org/10.1007/s10555-017-9707-8.

18. Huang J, Sun C, Su H et al. Bortezomib-Based Chemotherapy with Autologous Stem Cell Transplantation for Monoclonal Gammopathy of Renal Significance: A Case Report and Literature Review. Kidney Blood Press Res. 2019; 44(4): 858-69. http://doi.org/10.1159/000501314. Epub 2019.

19. Khera A, Panitsas F, Djebbari F et al. Long term outcomes in monoclonal gammopathy of renal significance. Br J Haematol. 2019; $186(5)$ : 706-16. http://doi.org/10.1111/bjh.15987. Epub 2019.

20. Leung N, Drosou ME, Nasr SH. Dysproteinemias and Glomerular Disease. Clin J Am Soc Nephrol. 2018; 13(1): 128-39. http://doi.org/10.2215/ CJN.00560117. Epub 2017.

21. Dima D, Dower J, Comenzo RL et al. Evaluating Daratumumab in the Treatment of Multiple Myeloma: Safety, Efficacy and Place in Therapy. Cancer Manag Res. 2020; 12: 7891-903. http://doi.org/10.2147/CMAR.S212526.

22. Kastritis $\mathrm{E}$, Theodorakakou $\mathrm{F}$, Roussou $\mathrm{M}$ et al. Daratumumab-based therapy for patients with monoclonal gammopathy of renal significance. Br J Haematol. 2020. http://doi.org/10.1111/bjh.17052. Epub ahead of print.

23. Leung N, Dingli D. Venetoclax in a Patient With Light Chain Deposition Disease Secondary to MGRS That Progressed After Kidney Transplantation Clin Lymphoma Myeloma Leuk. 2020; 20(8): e488-e91. http://doi.org/10.1016/j.clml.2020.03.013. Epub 2020.

24. Cibeira MT, Sanchorawala V, Seldin DC et al. Outcome of AL amyloidosis after high-dose melphalan and autologous stem cell transplantation: longterm results in a series of 421 patients. Blood. 2011; 118(16): 4346-52. http://doi.org/10.1182/blood-2011-01-330738. Epub 2011.

25. Palladini G, Kastritis E, Maurer MS et al. Daratumumab plus CyBorD for patients with newly diagnosed AL amyloidosis: safety run-in results of ANDROMEDA. Blood. 2020; 136(1): 71-80. http://doi.org/10.1182/blood.2019004460.

26. Nasr SH, Valeri AM, Cornell LD et al. Fibrillary glomerulonephritis: a report of 66 cases from a single institution. Clin J Am Soc Nephrol. 2011; $6(4)$ : 775-84. http://doi.org/10.2215/CJN.08300910. Epub 2011

27. Bridoux F, Hugue V, Coldefy $\mathrm{O}$ et al. Fibrillary glomerulonephritis and immunotactoid (microtubular) glomerulopathy are associated with distinct immunologic features. Kidney Int. 2002; 62(5): 1764-75. http://doi.org/10.1046/j.1523-1755.2002.00628.x.

28. Nasr SH, Markowitz GS, Stokes MB et al. Proliferative glomerulonephritis with monoclonal lgG deposits: a distinct entity mimicking immune-complex glomerulonephritis. Kidney Int. 2004; 65(1): 85-96. http://doi.org/10.1111/j.1523-1755.2004.00365.x.

29. Nasr SH, Satoskar A, Markowitz GS et al. Proliferative glomerulonephritis with monoclonal IgG deposits. J Am Soc Nephrol. 2009; 20(9): 2055-64. http://doi.org/10.1681/ASN.2009010110.

30. Gumber R, Cohen JB, Palmer MB et al. A clone-directed approach may improve diagnosis and treatment of proliferative glomerulonephritis with monoclonal immunoglobulin deposits. Kidney int. 2018; 94(1): 199-205.

31. Chauvet S, Frémeaux-Bacchi V, Petitprez F et al. Treatment of B-cell disorder improves renal outcome of patients with monoclonal gammopathy-associated C3 glomerulopathy. Blood. 2017; 129(11): 1437-47. http://doi.org/10.1182/blood-2016-08-737163. Epub 2017.

32. Zand L, Kattah A, Fervenza FC et al. C3 glomerulonephritis associated with monoclonal gammopathy: a case series. Am J Kidney Dis. 2013; 62(3): 506-14. http://doi.org/10.1053/j.ajkd.2013.02.370. Epub 2013.

33. Moog P, Jost PJ, Büttner-Herold M. Eculizumab as salvage therapy for recurrent monoclonal gammopathy-induced C3 glomerulopathy in a kidney allograft. BMC Nephrol. 2018; 19(1): 106. http://doi.org/10.1186/s12882-018-0904-7.

Authors' contributions: Grzegorz Kade: 25\%; Sebastian Spaleniak: 25\%; Janusz Hałka: 25\%; Stefan Antosiewicz: 25\%.

Conflict of interests:

None.

Financial support 